\title{
Dysfunctional Social Reinforcement Processing in Disruptive Behavior Disorders: An Functional Magnetic Resonance Imaging Study
}

\author{
Soonjo Hwang ${ }^{1}$, Harma Meffert ${ }^{2}$, Michelle R. VanTieghem ${ }^{3}$, Stephen Sinclair ${ }^{4}$, Susan Y. Bookheimer ${ }^{5}$, \\ Brigette Vaughan ${ }^{1}$, R. J. R. Blair ${ }^{2}$ \\ ${ }^{1}$ Department of Psychiatry, University of Nebraska Medical Center, Omaha, NE, ${ }^{2}$ Center for Neurobehavioral Research, Boys Town National \\ Research Hospital, Boys Town, NE, ${ }^{3}$ Department of Psychology, Columbia University, New York, NY, ${ }^{4}$ Department of Health and Human \\ Services, Section on Affective Cognitive Neuroscience, National Institute of Mental Health, National Institutes of Health, Bethesda, MD, \\ ${ }^{5}$ University of California and Brain Research Institute, Los Angeles, CA, USA
}

\begin{abstract}
Objective: Prior functional magnetic resonance imaging (fMRI) work has revealed that children/adolescents with disruptive behavior disorders (DBDs) show dysfunctional reward/non-reward processing of non-social reinforcements in the context of instrumental learning tasks. Neural responsiveness to socia/ reinforcements during instrumental learning, despite the importance of this for socialization, has not yet been previously investigated.

Methods: Twenty-nine healthy children/adolescents and 19 children/adolescents with DBDs performed the fMRI so$\mathrm{cial} /$ non-social reinforcement learning task. Participants responded to random fractal image stimuli and received social and non-social rewards/non-rewards according to their accuracy.

Results: Children/adolescents with DBDs showed significantly reduced responses within the caudate and posterior cingulate cortex (PCC) to non-social (financial) rewards and social non-rewards (the distress of others). Connectivity analyses revealed that children/adolescents with DBDs have decreased positive functional connectivity between the ventral striatum (VST) and the ventromedial prefrontal cortex (vmPFC) seeds and the lateral frontal cortex in response to reward relative to non-reward, irrespective of its sociality. In addition, they showed decreased positive connectivity between the vmPFC seed and the amygdala in response to non-reward relative to reward.

Conclusion: These data indicate compromised reinforcement processing of both non-social rewards and social non-rewards in children/adolescents with DBDs within core regions for instrumental learning and reinforcement-based decision-making (caudate and PCC). In addition, children/adolescents with DBDs show dysfunctional interactions between the VST, VMPFC, and lateral frontal cortex in response to rewarded instrumental actions potentially reflecting disruptions in attention to rewarded stimuli.
\end{abstract}

KEY WORDS: Disruptive behavior disorder; Social reward; Ventral striatum; Posterior cingulate cortex; Caudate; Ventro-medial prefrontal cortex.

\section{INTRODUCTION}

Conduct disorder (CD) and oppositional defiant disorder (ODD) are disruptive behavior disorders (DBDs) characterized by aggressive behavior, emotional dysregulation, and relationship difficulties. ${ }^{1)}$ Children and adoles-

Received: July 14, 2017 / Revised: September 12, 2017

Accepted: November 13, 2017

Address for correspondence: Soonjo Hwang, MD

985578 Nebraska Medical Center, Omaha, NE 68198-5578, USA

Tel: +1-402-552-6351, Fax: +1-402-552-6035

E-mail: soonjo.hwang@unmc.edu

ORCID: https://orcid.org/0000-0001-5117-2468 cents with DBDs demonstrate impairment on reinforcement-based decision-making tasks, ${ }^{2,3)}$ and considerable prior work relates observable behavioral impairments to dysfunction in brain regions critical for dysfunctional reward/non-reward/punishment processing. ${ }^{4,5)}$ Such impairment may further contribute to the difficulties in socialization for these children and adolescents. ${ }^{4,6)}$

Neural areas implicated in reinforcement processing are the ventromedial prefrontal cortex (vmPFC), ventral striatum (VST), anterior insula cortex (AIC) and posterior cingulate cortex (PCC). ${ }^{7-9)}$ These regions typically are known to show greater responsiveness to reward relative

(c) This is an Open-Access article distributed under the terms of the Creative Commons Attribution Non-Commercial License (http://creativecommons.org/licenses/by-nc/4.0) which permits unrestricted non-commercial use, distribution, and reproduction in any medium, provided the original work is properly cited. 
to punishment and non-rewards across various tasks, ${ }^{7,8)}$ and there are indications that they are similarly responsive to non-social (money gain/loss) and social reinforcements (happy/sad expressions). ${ }^{10)}$ The amygdala may also be implicated particularly for social punishments/non-rewards (distress of others). ${ }^{11,12)}$ Additionally, regions implicated in response control (dorsomedial frontal cortex, AIC/inferior frontal gyrus and caudate) have been implicated in the avoidance of sub-optimal choices. ${ }^{3,13-16)}$

Appropriate processing of social reward is critical for adolescents' normal psychosocial development, ${ }^{17,18)}$ and emotional displays (e.g., of sadness or fear) can be modifiers of observer behavior. ${ }^{19-21)}$ Children/adolescents with DBDs show disrupted reinforcement processing within the striatum and vmPFC. 2,3,5,22-25) Previous studies have demonstrated that children/adolescents with DBDs, particularly those with elevated callous-unemotional traits, display impaired recognition of emotional expression, particularly sadness and fear, ${ }^{26-29)}$ and a series of studies has revealed reduced amygdala responses to distress cues (sad and fearful expressions). ${ }^{28,30,31)}$ This work, however, has not examined socia/reinforcement processing, and to our knowledge, no previous work has addressed the extent to which these children/adolescents demonstrate disruption in socia/ reinforcement-based decision-making.

The goal of the current paper is to examine decision-making in children/adolescents with DBDs on the basis of non-social and social reinforcements using a task developed by Scott-Van Zeeland et al. ${ }^{22)}$ in 2010. Given CD and ODD share many symptoms, ${ }^{1)}$ particularly aggressive behavior, the purpose of this study is to identify the neural areas and their dysfunction related to symptom manifestation across these disorders. In line with the National Institutes of Health (NIH)-initiated research domain criteria (RDoC) approach to understanding mental health disorders, this is a departure from a diagnosis-based approach to a mechanism-driven understanding of the pathophysiology of DBD. ${ }^{32,33)}$

Based on previous work, ${ }^{2,4,22-24,30,31)}$ we hypothesize that children/adolescents with DBDs, relative to comparison children/adolescents, would show reduced: (i) differential recruitment of the vmPFC, PCC and striatum to rewards compared to non-rewards (social and non-social); (ii) reduced responses to social non-rewards (sad facial expressions) within the amygdala; and (iii) reduced positive connectivity between core regions involved in re- ward processing (vmPFC, VST) and cortical regions involved in reinforcement processing and attention (amygdala, PCC, lateral frontal and parietal cortices).

\section{METHODS}

\section{Subjects}

Fifty-six children and adolescents participated: 30 healthy and 26 with DBDs (age, 12-18 years). Children and adolescents were recruited from the community via newspaper ads, fliers, and referrals from area mental health practitioners. Eight participants ( 1 healthy, 7 with DBDs) were not included in the final data analysis because of their excessive movement; repetition times (TRs) were censored if movement $>1 \mathrm{~mm}$ within the TR and the participant was excluded if this occurred for $>15 \%$ of trials. In the final analysis, 48 subjects were included after this process (29 healthy and 19 DBDs). There were no group differences in age, gender, handedness, or intelligence quotient (IQ) (Table 1). Written informed consent for participation in the study was obtained from all subjects' legal guardians and assent from the children/adolescents. Ethics approval for this study was granted by the $\mathrm{NIH}$ Combined Neurosciences Institutional Review Board under protocol number 05-M-0105.

Table 1. Characteristics of healthy children/adolescents and children/ adolescents with DBDs

\begin{tabular}{lccc}
\hline \multicolumn{1}{c}{ Demographic } & $\begin{array}{c}\text { Healthy } \\
\text { children/adoles } \\
\text { cents }(\mathrm{n}=29)\end{array}$ & $\begin{array}{c}\text { Children/adole } \\
\text { scents with } \\
\text { DBDs }(\mathrm{n}=19)\end{array}$ & $\begin{array}{c}p \text { value } \\
(\mathrm{df})\end{array}$ \\
\hline Age (yr) & $14.21 \pm 1.90$ & $14.79 \pm 2.04$ & $0.318(1)$ \\
QQ & $105.28 \pm 11.38$ & $100.16 \pm 11.09$ & $0.131(1)$ \\
Gender (male/female) & $13 / 16$ & $13 / 6$ & $0.095(1)$ \\
Handedness (left/right) & $4 / 25$ & $2 / 17$ & $0.554(1)$ \\
DBD & 0 & 19 & \\
CD & 0 & 9 & \\
ODD & 0 & 2 & \\
CD/ADHD & 0 & 3 & \\
ODD/ADHD & 0 & 3 & \\
CD/SA & 0 & 2 & \\
Medication & 0 & $3 *$ & \\
\hline
\end{tabular}

Values are presented as mean \pm standard deviation or number only. $\mathrm{DBD}$, disruptive behavior disorder; $\mathrm{df}$, degree of freedom; IQ, intelligence quotient; $\mathrm{CD}$, conduct disorder; $\mathrm{ODD}$, oppositional defiant disorder; $\mathrm{ADHD}$, comorbidity of attention deficit hyperactivity disorder; SA, comorbidity of substance abuse (cannabinoid).

*1: amphetamine+risperidone; 1: amphetamine; 1: methylphenidate+quetiapine. 
All children and adolescents completed the Kiddie Schedule for Affective Disorders and Schizophrenia (K-SADS). ${ }^{34)}$ The diagnoses of DBD were made by an expert child and adolescent psychiatrist on the basis of the K-SADS (including the attention deficit hyperactivity disorder [ADHD], CD and ODD modules) performed by a doctoral-level clinical psychologist. Healthy children and adolescents presented without current or past psychiatric diagnoses. The K-SADS has showed good validity as well as good inter-rater reliability (kappa $>0.75$ for all diagnoses). ${ }^{34)}$ Exclusion criteria for all participants were autism spectrum disorders, Tourette's syndrome, bipolar disorder, lifetime history of psychosis, depression, generalized, social or separation anxiety disorder, post-traumatic stress disorder, neurologic disorder including seizure or epilepsy, history of major head trauma including skull fracture, substance dependence (substance abuse was not exclusionary), major medical illness, and IQ of $<80$. IQ was assessed with the Wechsler Abbreviated Scale of Intelligence 2-subtest form; ${ }^{35)}$ There were no significant group differences in IQ (Table 1).

\section{Experimental Design}

An adapted version of the social and non-social (monetary) reinforcement-learning task was used for the study. ${ }^{18)}$ On each trial of a run (Fig. 1), subjects were presented with a fractal image (duration, 2,000 ms) and were asked to categorize it into one of two groups by button press. The participants received the following instructions: "You are going to play a computer game. In this game, you will see colorful circles. You need to find out if the picture belongs to group 1 or 2 . You can do this by pressing the buttons when you see the picture. Of course you will not know in the beginning which picture belongs to which group. However, you will be given feedback after each response, to tell you whether you are right or wrong. Thus as the game goes on, you will find out which picture belongs to which groups".

The fractal image was followed by an inter-stimulus interval of randomly jittered length (500-1,500 ms) during which a blank screen was presented. After this, the subject received feedback (duration, 1,250 ms). Following the feedback, there was then an inter-stimulus interval of randomly jittered length (1,250-2,500 ms) during which a blank screen was presented before the next trial began when another fractal image was presented.
Each task was comprised of two social (facial expressions) and two non-social (monetary) reinforcement runs. During non-social (monetary) runs, correct responses were reinforced with the image of a 5-dollar bill and the words "That's right!" (monetary reward) and incorrect responses with the image of a 5-dollar bill struck through with red lines and the words "That's wrong" (monetary non-reward). On neutral trials, feedback consisted only of the words "That's right", or "That's wrong", depending on the accuracy of the participants' response (monetary neutral). The participants did not receive the actual money gained. During social runs, correct responses were reinforced with a happy face and the words "That's right!" (social reward) and incorrect responses with the image of a sad face and the words "That's wrong" (social non-reward). On neutral trials, feedback consisted of a neutral face and the words "That's right", or "That's wrong" depending on the accuracy of the participant's response (social neutral). The pictures used for social feedback (the happy, neutral and sad expressions) were taken from a single individual, and each picture had clearly distinctive valence (Fig. 1).

Each run (social or non-social [monetary]) contained

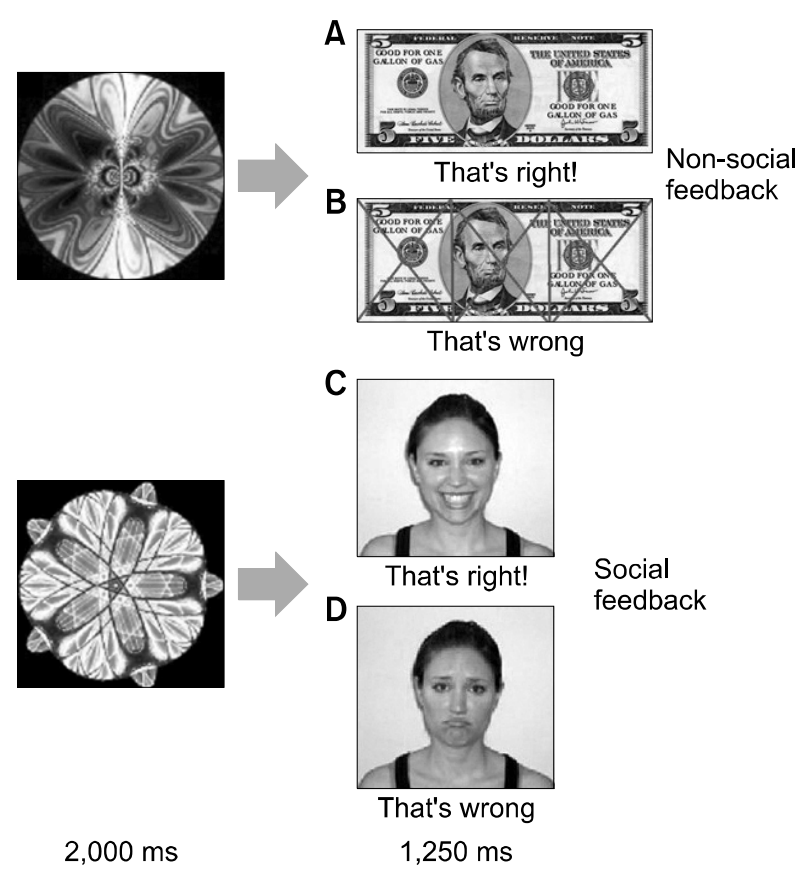

Fig. 1. Example trial sequences. (A) Non-social reward, (B) non-social non-reward, (C) social reward, (D) social non-reward.

Adapted from the article of Hwang et al. (Clin Psychopharmacol Neurosci 2017;15:369-381). 
six fractal images. Social runs involved a different set of fractal images from those used in non-social runs. Four of the 6 fractals always provided information with respect to whether the participant's response was correct or incorrect with $100 \%$ probability. However, for $67 \%$ of responses to these trials, feedback was social or non-social (depending on the run) while for the other $33 \%$ of responses to these trials feedback was neutral. The other 2 of the six fractals were reinforced at chance level, i.e. these fractals were randomly rewarded in $50 \%$ of the trials, irrespective of the participants' response. Participants completed four runs in total and the presentation order of the runs was counterbalanced across the participants (social-monetary-social-monetary or monetary-socialmonetary-social). Each run involved 54 trials (9 presentations of each of the 6 fractal images).

\section{Image Acquisition and Analysis}

Whole-brain blood oxygen level-dependent (BOLD) functional magnetic resonance imaging (fMRI) data were acquired using a 3-T MRI scanner (General Electronics, Chicago, IL, USA). Following sagittal localization, functional $\mathrm{T}^{*}$-weighted images were acquired using an echo-planar single-shot gradient echo pulse sequence with a matrix of $64 \times 64 \mathrm{~mm}$, TR of 3,000 ms, echo time (TE) of $30 \mathrm{~ms}$, field of view (FOV) of $240 \mathrm{~mm}$, and voxels of $3.75 \times 3.75 \times 4 \mathrm{~mm}$. Images were acquired in 30 continuous $4 \mathrm{~mm}$ axial slices per brain volume across four runs. The duration of each run was 6 minutes 40 seconds. In the same session, a high-resolution T1-weighed anatomical image was acquired to aid with spatial normalization (three-dimensional Spoiled GRASS; TR=8.1 ms; $\mathrm{TE}=3.2 \mathrm{~ms}$, flip angle $20^{\circ} ; \mathrm{FOV}=240 \mathrm{~mm}, 128$ axial slices, thickness $=1.0 \mathrm{~mm} ; 256 \times 256$ acquisition matrix).

\section{fMRI Analysis}

Data were analyzed within the framework of a random effects general linear model using Analysis of Functional Neuroimages (AFNI). Both individual and group-level analyses were conducted. The first 5 volumes in each scan series, collected before equilibrium magnetization was reached, were discarded. Motion correction was performed by registering all volumes in the Environmental Performance Index (EPI) dataset to a volume that was collected shortly before acquisition of the high-resolution anatomical dataset.
The EPI datasets for each subject were spatially smoothed (using an isotropic $6 \mathrm{~mm}$ Gaussian kernel) to reduce the influence of anatomical variability among the individual maps in generating group maps. Next, the time series data were normalized by dividing the signal intensity of a voxel at each time point by the mean signal intensity of that voxel for each run and multiplying the result by 100 . Resultant regression coefficients represented a percent signal change from the mean. The model involved six motion regressors, four regressors for stimulus onset (onset of fractal images for non-social reinforcement with $100 \%$ probability, social reinforcement with $100 \%$ probability, non-social reinforcement with $50 \%$ probability, social reinforcement with $50 \%$ probability), and the following task regressors: non-social reward, non-social non-reward, non-social neutral, social reward, social non-reward, and social neutral. A regressor modeling incorrect responses was also included. All regressors were convolved with a canonical hemodynamic response function (HRF) to account for the slow hemodynamic response (with time point commencing at time of first image onset). There was no significant regressor collinearity. Linear regression modeling was performed using the 11 regressors described earlier, plus regressors to model a first-order baseline drift function. This produced $\beta$ coefficients and associated $t$ statistics for each voxel and regressor.

After excluding subjects with excessive movements (defined as $>15 \%$ TRs discarded due to movement $>1$ $\mathrm{mm}$ ), there were no significant group differences in movement parameters (average movement across each time point) including delta-roll, delta-pitch, delta-yaw, delta-ds, delta-dp, and delta-dl; $F(1,45)=1.23-2.31, p>0.5$.

The participants' anatomical scans were individually registered to the Talairach and Tournoux atlas. ${ }^{36)}$ The individuals' functional EPI data were then registered to their Talairach anatomical scan within AFNI. Linear regression modeling was performed using the 6 regressors described earlier, plus regressors to model a third-order baseline drift function. This produced $\beta$ coefficients and associated $t$ statistics for each voxel and regressor.

The BOLD data were analyzed via a 2 (diagnosis: healthy children/adolescents, children/adolescents with DBDs) by 2 (reinforcement: reward, non-reward) by 2 (sociality of feedback: non-social, social) 3dMVM ANOVA. The neutral feedback trials were not included in 
the group level ANOVA analyses, due to the concern of potential power loss related to an additional level to the reinforcement factor. We considered statistical maps for each main effect and interaction by thresholding at a single-voxel $p$ value of $<0.005$. With these maps, a result was considered significant if it was predicted a priori and had an extent threshold greater than 10 voxels. A priori regions (vmPFC, PCC, VST, middle frontal cortex, and caudate) were selected from the following criteria: (1) neural areas involved reinforcement processing by the previous studies, and (2) neural areas showing dysfunction in children/adolescents with DBD. ${ }^{2,7-9,22,37)}$ It is worth considering recent suggestions that a more conservative approach that strictly controls for type I error should be adopted. ${ }^{38)}$ This approach contrasts with arguments that such a strict approach fails to account for theory-driven hypotheses and introduces an unacceptable amount of type II error Lieberman et al., ${ }^{37)}$ under review;. The disadvantage of the conservative approach is that there are no post-publication remedies for type II error. Data is simply not available for later consideration. In contrast, results that are type I errors will fail to replicate and/or will not survive meta-analysis. Furthermore, in order to facilitate future meta-analytic work, effect sizes ( $\eta 2$ ) for all clusters and follow-up $t$ tests (partial eta $[\eta]$ square) are reported.

\section{Generalized form of Context-dependent Psychophysiological Interaction (gPPI) Analysis}

The gPPI analyses were conducted to examine differences in functional connectivity between healthy children/adolescents and children/adolescents with DBDs, following the method described by McLaren et al. ${ }^{39)}$ Our main goal was to examine differences between these two groups in functional connectivity within the reinforcement processing network. As such, we took two seed regions identified from the BOLD response ANOVA (main effect of reinforcement using an initial threshold of $p<$ $0.001)$; this $p$ value was stricter than that used for the BOLD response analyses to ensure the identification of a relatively small seed that was within the one anatomical region of interest (ROI): right $\mathrm{VmPFC}$ (coordinates: 1.5, 49.5, 2.5; 32 voxels) and right VST (coordinates: 13.5, 7.5, - 3.5; 36 voxels) (Supplementary Fig. 1). These regions met our two criteria for ROI selection. First, they were revealed via the main effect of reinforcement in the main BOLD response ANOVA. As such, they were re- gions specifically sensitive to reinforcement across different groups (a ROI identified through a diagnosis-by-reinforcement interaction might have revealed differences via gPPI that simply reflected reduced signal in the region in one of the groups, most likely children/adolescents with DBD). Second, they were regions identified to be reward sensitive within the previous literature. ${ }^{7,9)}$

The average activation from these seed regions was extracted from the preprocessed time-series as used in the main analysis, but before the spatial smoothing had been applied. The seed time-series was first detrended and deconvolved. Eleven interaction terms were created by multiplying the detrended and deconvolved seed timeseries with eleven indicator regressors, which indicated the onset of the four stimulus onset, six feedback types (one for each reinforcement and sociality condition), and one incorrect responses. Finally, these eleven interaction terms were convolved with the HRF to create eleven gPPI regressors. Linear regression modeling was performed using the task regressors from the main analysis, six motion regressors, a regressor reflecting the seed time-series, the eleven gPPI regressors and regressors to model a first-order baseline drift function. This produced a $\beta$ coefficient and associated $t$ statistics for each voxel and regressor. A 2 (diagnosis: healthy children/adolescents, children/adolescents with DBDs) by 2 (reinforcement: reward, non-reward) by 2 (sociality of feedback: non-social, social) ANOVA was then applied to the data. In addition to this, based on the previous studies showing disruptive connectivity between vmPFC and amygdala in children/adolescents with DBD, ${ }^{28,40,41)}$ a right amygdala ROI was selected for the gPPI analysis, using an anatomically defined mask (Eickhoff-Zilles architectonic atlas: 50\% probability), ${ }^{36)}$ yielded a threshold of $162 \mathrm{~mm}^{3}$ at an initial significance threshold of 0.02. Follow-up analyses were performed to facilitated interpretations. For these analyses, average percent signal change was measured across all voxels within the amygdala ROI generated from the functional masks, and data were analyzed using appropriate follow-up independent $t$ tests within SPSS ver. 22.0 (IBM Co., Armonk, NY, USA).

Note that while the BOLD response and connectivity analyses can be considered relatively independent, the use of two separate seeds for the gPPI analyses does lead to experiment-wise alpha inflation. Consequently, the gPPI analyses should be considered exploratory. 


\section{RESULTS}

\section{Behavioral Data}

Two 2 (diagnosis: healthy children/adolescents, children/adolescents with DBDs) by 2 (sociality of feedback: non-social, social) by 2 (phase: first phase [first two runs], second phase [last two runs]) ANOVAs were applied to the reaction time (RT) and accuracy data respectively (Supplementary Table 1). With respect to accuracy data, there was a significant main effect of phase $(F(1,46)=$ 14.93, $p<0.001)$, as well as a significant diagnosisby-phase interaction $(F(1,49)=5.78, p=0.02)$. Specifically, accuracy was significantly better in the last two runs relative to the first two runs $(\mathrm{t}(47)=4.25, p<0.001)$. Moreover, healthy children/adolescents showed significantly greater accuracy than children/adolescents with DBD in the second $(\mathrm{t}(46)=2.03, p<0.05)$, but not the first phase of the study $(\mathrm{t}(46)=0.44, p=0.66)$. No other main effects or interactions were significant.

With respect to RT data, there was a significant main effect of phase $(F(46)=29.90, p<0.001)$. Specifically, RTs were significantly shorter in the last two runs relative to the first two runs $(t(47)=5.68, p<0.001)$. These revealed no other significant main effects or interactions.

\section{fMRI Data}

A 2 (diagnosis: healthy children/adolescents, children/ adolescents with DBDs) by 2 (reinforcement: reward, non-reward) by 2 (sociality: non-social feedback, social feedback) ANOVA was applied to the BOLD data. This revealed regions showing significant main effects of reinforcement (vmPFC and bilateral VST [centered in right
VST but extending to the left hemisphere]) (Table 2). Within these regions, BOLD responses were significantly greater to reward than non-reward. These became the seeds for our gPPI analyses. In addition, the ANOVA revealed regions showing a main effect of sociality as well as regions showing significant reinforcement-by-sociality interactions (Supplementary Section 1-4). Importantly, this ANOVA revealed the following key findings.

\section{Diagnosis-by-reinforcement-by-sociality interaction}

Regions showing diagnosis-by-reinforcement-by-sociality interactions included right PCC (Fig. 2A) and left caudate (Fig. 2B); see Table 2. Within these regions, post-hoc analysis showed that groups differed significantly in their response to social relative to monetary rewards versus non-rewards $(\mathrm{t}(46)=3.47$ and $3.15, p=$ 0.001 and $0.03, \eta 2=0.21$ and 0.26 for PCC and caudate respectively). Healthy children showed significantly greater responses to non-social rewards relative to social rewards (money won vs. happy facial expressions) relative to social non-rewards (sad facial expression) relative to non-social non-rewards (no money won) than children/adolescents with DBDs; i.e., healthy children showed greater responses to [(monetary reward - social reward) (monetary non-reward-social non-reward)] relative to children with DBD (see Fig. 2 for significant contrasts). Indeed, while healthy children/adolescents showed significantly greater responses to non-social relative to social rewards and social relative to non-social non-rewards, children/adolescents with DBDs showed greater responses to social relative to non-social rewards but greater responses non-social relative to social non-rewards.

Table 2. Brain Regions showing significant diagnosis-by-reinforcement-by-sociality interaction

\begin{tabular}{|c|c|c|c|c|c|c|c|c|}
\hline \multirow{2}{*}{ Region* } & \multicolumn{5}{|c|}{ Coordinates of peak activation } & \multirow{2}{*}{$\mathrm{F}^{\dagger}$} & \multirow{2}{*}{ Voxel } & \multirow{2}{*}{ Partial $\eta 2$} \\
\hline & Left/right & $\mathrm{BA}$ & $\mathrm{x}$ & y & z & & & \\
\hline \multicolumn{9}{|l|}{ Main effect of reinforcement } \\
\hline Ventromedial prefrontal cortex & Right & 10 & 1.5 & 49.5 & 2.5 & 24.35 & 118 & 0.39 \\
\hline Ventral striatum & Right & & 13.5 & 7.5 & -3.5 & 34.58 & 186 & 0.55 \\
\hline \multicolumn{9}{|l|}{ Main effect of diagnosis } \\
\hline Posterior cingulate cortex & Left & 30 & -7.5 & 49.5 & 23.5 & 22.69 & 187 & 0.47 \\
\hline \multicolumn{9}{|c|}{ Diagnosis by reinforcement by sociality } \\
\hline Posterior cingulate cortex & Right & 31 & 10.5 & -37.5 & 35.5 & 11.95 & 22 & 0.21 \\
\hline Caudate & Left & & -1.5 & 1.5 & 14.5 & 14.11 & 20 & 0.26 \\
\hline
\end{tabular}

BA, Broadmann area.

${ }^{*}$ According to the Talairach Daemon Atlas (http://www.nitrc.org/projects/tal-daemon); ${ }^{\dagger}$ Degree of freedom=38. $p<0.005$. 

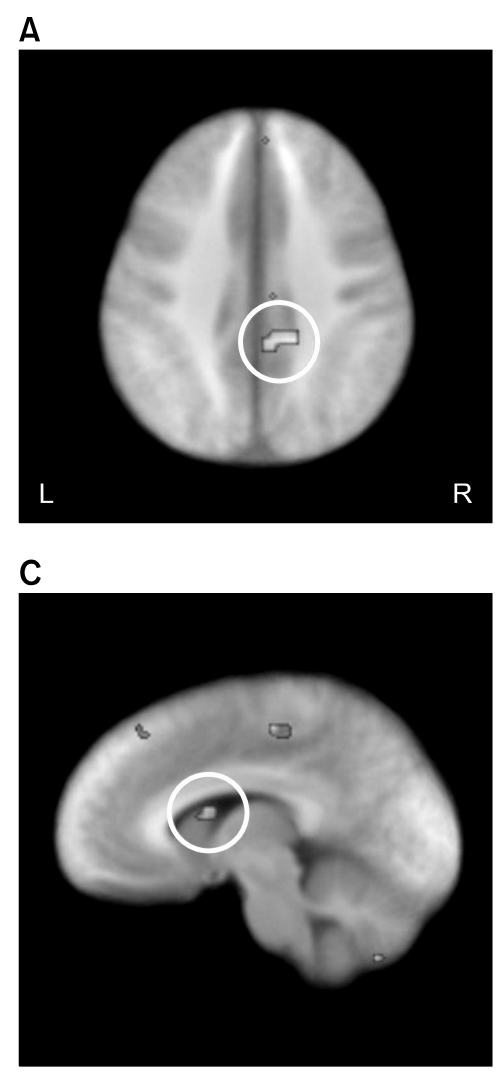

B

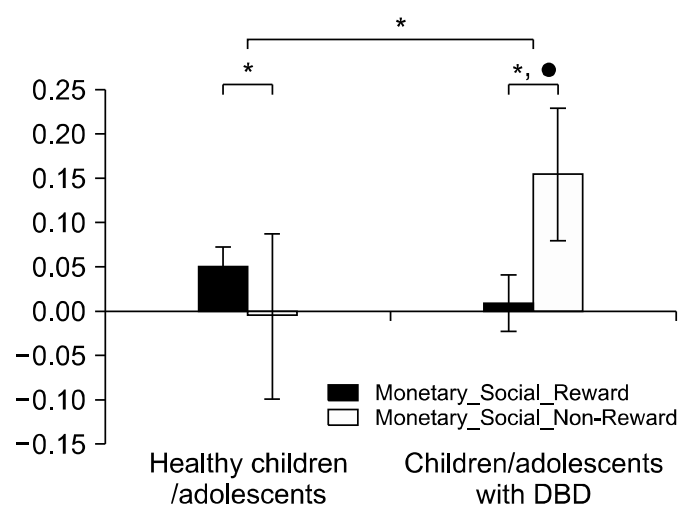

D

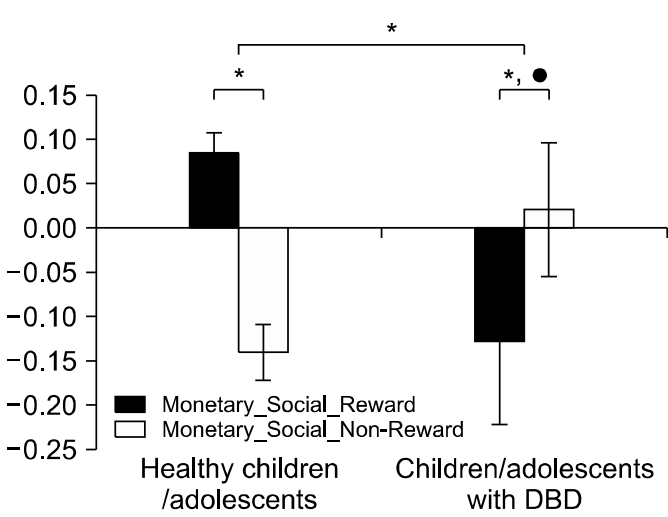

Fig. 2. Regions showing significant diagnosis-by-reinforcement-by-soci ality interaction. (A) Right posterior cingulate cortex (coordinates: 10.5, $-37.5,35.5)$ (B) Parameter estimates for posterior cingulate cortex. (C) Left caudate (coordinates: $-1.5,1.5$, 14.5). (D) Parameter estimate for caudate region of interest.

DBD, disruptive behavior disorders; Monetary_Social_Reward, social reward relative to moneatry reward; Monetary_Social_Non-reward, social non-reward relative to monetary non-reward.

*Statistically significant; $\bullet$, as a trend.

Table 3. Brain regions showing a significant interaction of connectivity between healthy children/adolescents and children/adolescents with DBDs

\begin{tabular}{|c|c|c|c|c|c|c|c|c|}
\hline \multirow{2}{*}{ Region* } & \multicolumn{5}{|c|}{ Coordinates of peak activation } & \multirow{2}{*}{$\mathrm{F}^{\dagger}$} & \multirow{2}{*}{ Voxels } & \multirow{2}{*}{ Partial $\eta 2$} \\
\hline & Left/right & $\mathrm{BA}$ & $x$ & $y$ & z & & & \\
\hline \multicolumn{9}{|c|}{$\begin{array}{l}\text { (A) Right ventromedial prefrontal cortex seed ( } p=0.001,32 \text { voxels) } \\
\text { Diagnosis by reinforcement }\end{array}$} \\
\hline Middle frontal gyrus & Left & 46 & -43.5 & 28.5 & 23.5 & 14.68 & 45 & 0.30 \\
\hline Amygdala ROI & Right & & 16.5 & -1.5 & 24.5 & 15.46 & 30 & 0.31 \\
\hline \multicolumn{9}{|c|}{$\begin{array}{l}\text { (B) Right ventral striatum seed ( } p=0.0001,36 \text { voxels) } \\
\text { Diagnosis-by-reinforcement }\end{array}$} \\
\hline Middle frontal gyrus & Right & 46 & 46.5 & 28.5 & 20.5 & 22.31 & 53 & 0.37 \\
\hline
\end{tabular}

BA, Broadmann area; ROI, regions of interest.

${ }^{*}$ According to the Talairach Daemon Atlas (http://www.nitrc.org/projects/tal-daemon); ${ }^{\dagger}$ Degree of freedom $=38$. $p<0.005$ except (A) and (B) ( $p$ value specified for those areas).

\section{Main effect of diagnosis}

A region within the left PCC showed a significant main effect of diagnosis (Table 2). Within this region, post-hoc analysis showed BOLD responses were significantly greater for healthy children/adolescents to reinforcement information compared to children/adolescents with DBDs.

\section{MRI Results: gPPI Results}

Two 2 (diagnosis)-by-2 (reinforcement: reward, non-re- ward)-by-2(sociality: social, non-social) ANOVAs were conducted on the gPPI data using seeds identified via the main effect of reinforcement (i.e., right VST and right vmPFC). Results relevant to predictions are considered below. For full results, see Table 3.

\section{Right VST seed}

A lateral region of right middle frontal gyrus showed a significant diagnosis-by-reinforcement interaction (Table 


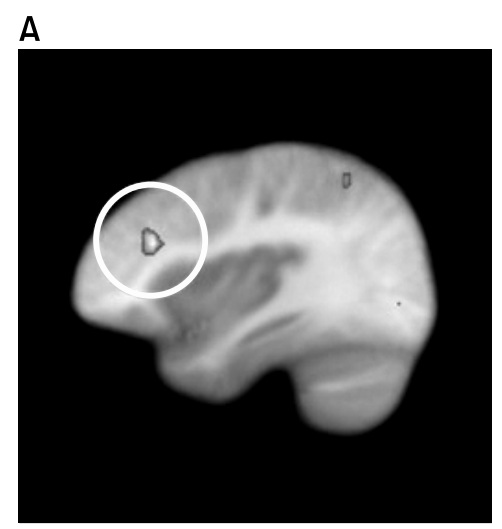

B
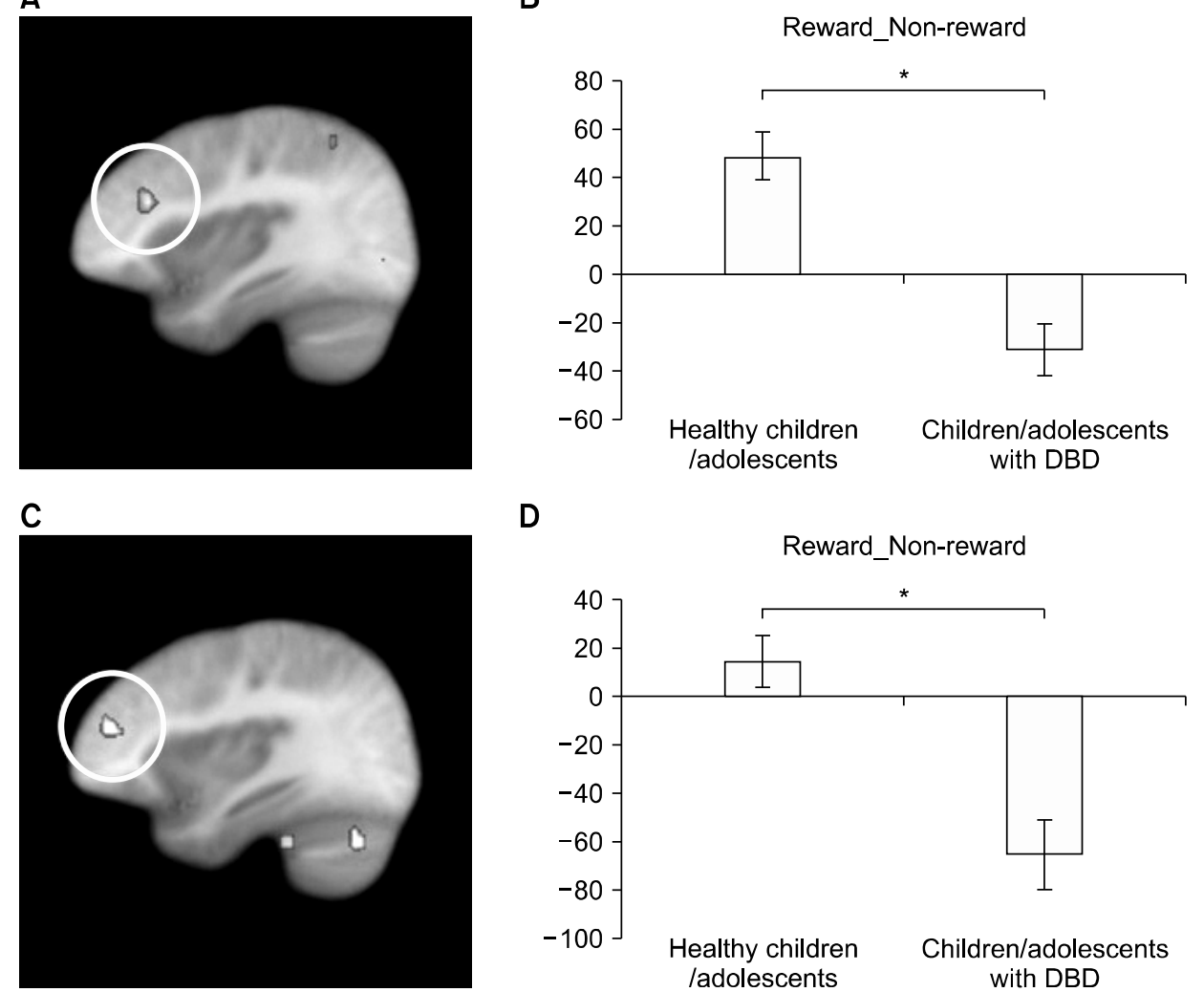

D
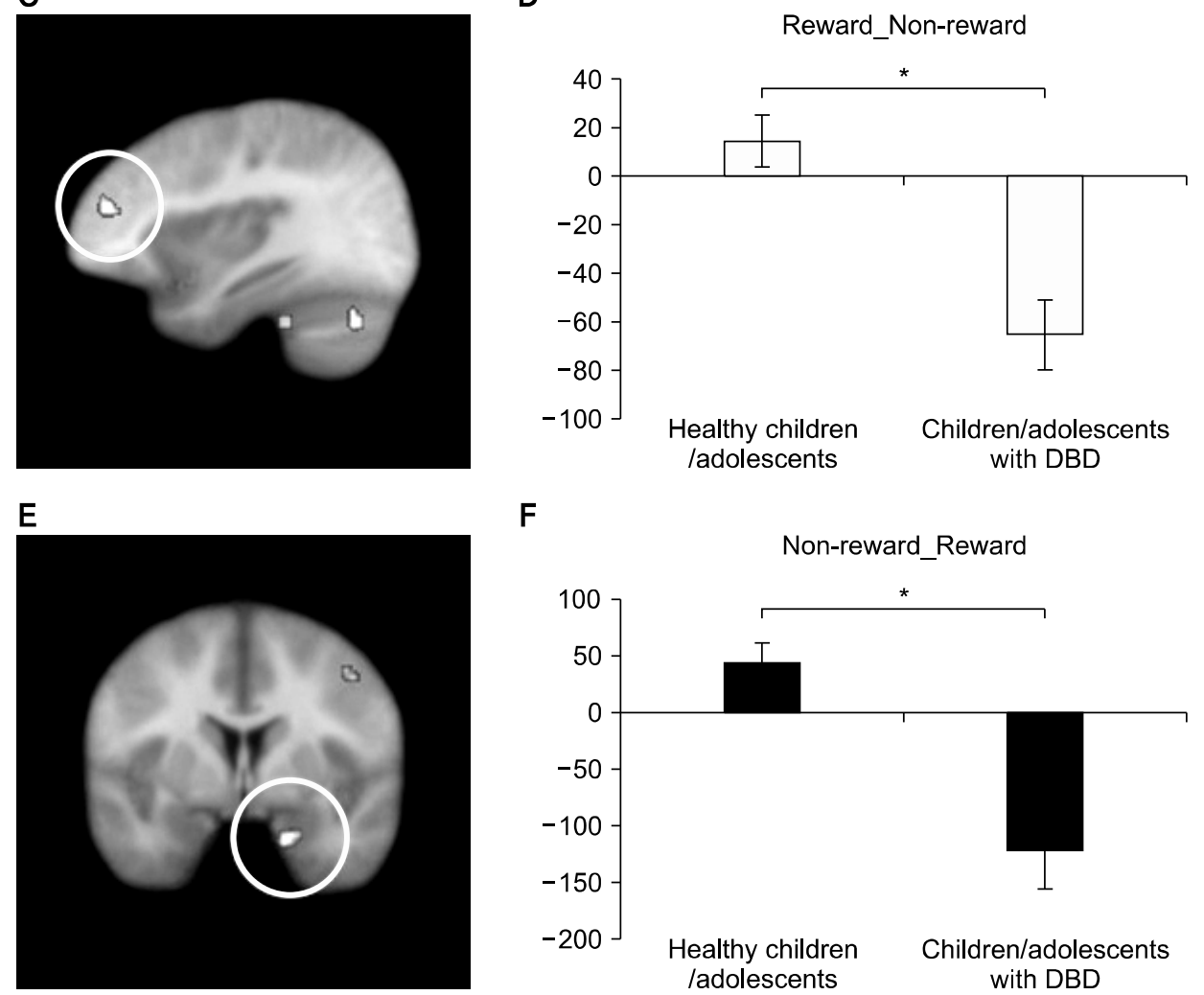

F

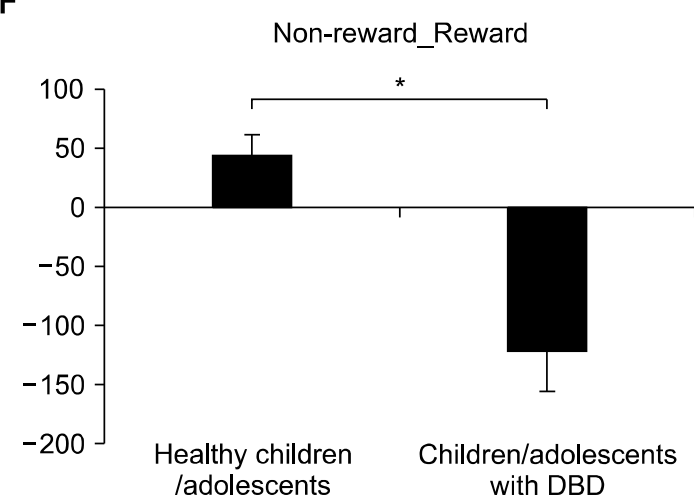

Fig. 3. Regions showing significant diagnosis-by-reinforcement interaction of connectivity with right ventral striatum seed on generalized form of context-dependent psychophysiological interaction (gPPI) analysis. (A) Right middle frontal gyrus (coordinates: 46.5, 28.5, 20.5). (B) Parameter estimates for right middle frontal gyrus; regions showing significant diagnosis-by-reinforcement interaction connectivity with left ventromedial prefrontal cortex seed on gPPI analysis. (C) Right middle frontal gyrus (coordinates: 52.5, 4.5, 35.5). (D) Parameter estimates for right middle frontal gyrus. (E) Left amygdala region of interest (ROI) (coordinates: 16.5, - 1.5, 24.5), (F) Parameter estimates for left amygdala ROI.

Reward_Non-reward, reward relative to non-reward; Non-reward_Reward, non-reward relative to reward; DBD, disruptive behavior disorders. *Statistically significant.

3). Follow-up analyses revealed that children/adolescents with DBDs showed significantly less positive connectivity with the right VST seed in response to reward relative to non-reward, compared to healthy children/adolescents $(\mathrm{t}(46)=26.55, p<0.001, \eta 2=0.37)$ (Fig. 3A, 3C).

\section{vmPFC seed}

Regions showing significant diagnosis-by-reinforcement interactions were observed within left middle frontal gyrus and the right amygdala ROI (Table 2). Follow-up analyses on BOLD responses revealed that for middle frontal gyrus, children/adolescents with DBDs showed significantly less positive connectivity with the vmPFC in response to reward relative to non-reward, compared to healthy children $(\mathrm{t}(46)=19.78, p<0.001, \eta 2=0.30)$-and instead actually showed negative connectivity between these regions. In the right amygdala ROI, children/adolescents with DBDs showed significantly less positive connectivity with the vmPFC seed in response to non-reward relative to reward, compared to healthy children/adolescents $(\mathrm{t}(46)=20.42, p<0.001, \eta 2=0.31)$ (Fig. 3E). 


\section{Potential Confounds}

We conducted analyses excluding (1) children/adolescents with DBDs on psychotropic medications, (2) children/adolescents with DBDs and substance abuse (2 subjects, both using cannabinoid for unknown duration), and (3) children/adolescents with DBDs and comorbid ADHD. Participants treated with psychostimulants were advised to hold medication for at least 48 hours prior to MRI scans. These analyses revealed similar results to the main analysis reported above (Supplementary Material). We also measured the callous-unemotional trait using the Inventory of Callous-Unemotional Trait (ICU); ${ }^{42)}$ however, no correlation with BOLD responses was observed.

\section{DISCUSSION}

The current study investigated neural correlates of decision-making in children/adolescents with DBDs as a function of both non-social (money gain/no gain) and social (happy/sad faces) reinforcements. There were three main findings: First, healthy children showed significantly greater responses within the caudate and PCC to non-social rewards (money won) relative to social rewards (happy facial expressions) relative to social non-rewards (money loss) relative to non-social non-rewards (sad facial expression) than children/adolescents with DBDs. Second, children/adolescents with DBDs showed significantly less positive connectivity between seeds within both the VST and VmPFC, and the lateral regions of middle frontal gyrii in response to rewards relative to non-rewards, compared to healthy children/adolescents. Third, children/adolescents with DBDs, compared to healthy children/adolescents, showed significantly less positive connectivity between the vmPFC and the amygdala ROI in response to non-rewards (social and non-social).

Previous studies have reported that children/adolescents with DBDs show reduced responses to reinforcement information within the striatum and vmPFC. ${ }^{2,3,5,22-25)}$ On this basis and given findings that both regions are responsive to social and non-social rewards for a meta-analysis, ${ }^{43)}$ we hypothesized that children/adolescents with DBDs would show reduced responses within these regions to social and non-social rewards relative to social and non-social non-rewards compared to healthy children/adolescents. This prediction, however, was not confirmed, and it was not due to a lack of signal within either region as both the
vmPFC and VST showed clear main effects of reinforcement. Participants showed greater responses within these regions to rewards relative to non-rewards regardless of sociality and whether or not the participant presented with DBDs.

Group differences emerged in regions showing differential responsiveness to social rewards relative to non-social rewards and non-rewards. Notably, children/adolescents with DBDs showed a particularly reduced responses to non-social rewards in a region of the dorsal striatum/caudate that healthy children/adolescents particularly recruited for these rewards. The dorsal striatum/ caudate is critically involved in reinforcement processing, particularly in the context of instrumental learning tasks, ${ }^{44,45)}$ and impairments in the striatal response to reward (always non-social reward in previous studies) in children/adolescents with DBDs have been mostly confined to dorsal striatum/caudate ${ }^{2,3,22,23,25)}$; though see Wrase et al. ${ }^{46)}$ The current study replicated previous work indicating reduced reward processing in the caudate in children/adolescents with DBDs during instrumental learning tasks. This is likely to lead to poor decision-making and maladaptive behavior due to difficulty in processing salient reward information in this population. ${ }^{4)}$ Our results extend earlier work by showing that the impairment in reward processing is confined to non-social rewards as this study showed no significant differences in the response within the caudate (or VST or vmPFC) to social rewards but significant group differences within the caudate to non-social rewards. This is in noted contrast to children/adolescents with autism who have been reported to show reduced ventral striatal responses to social rewards on this task. ${ }^{18)}$

Previous studies have reported that children/adolescents with DBDs show reduced responses to social nonrewards (distress cues/sad or fearful faces). Children/adolescents with DBDs show reduced autonomic responses $^{47)}$ and amygdala responses to sad facial expressions $^{48)}$ as well as reduced amygdala responses to fearful expressions. ${ }^{28)}$ On this basis, we had predicted that children/adolescents with DBDs would show reduced responses to social non-rewards (sad facial expression). This prediction was partially confirmed. There were no group differences in amygdala responses to social non-rewards, however, within the right PCC children/adolescents with DBDs showed significantly less BOLD responses than did 
the healthy children/adolescents. Given the role of the PCC in the representation of subjective value, ${ }^{7)}$ this result might represent a failure to represent the value of the distress of others by children/adolescents with DBDs. It has been argued that individuals with DBDs may process the distress of others to a lesser extent and thus be less likely to change their behavior as a consequence of it. ${ }^{49,50)}$ It should be noted that the vmPFC is also implicated in the representation of subjective value but no group differences in responsiveness were observed within this region.

Our third prediction was that children/adolescents with DBDs would show reduced connectivity between core regions involved in reward processing (vmPFC, VST) and cortical regions implicated in reinforcement processing and attention. Although children/adolescents with DBDs and healthy children/adolescents did not significantly differ in their responsiveness within the VST and VmPFC to reward relative to non-reward information, there were, significant group differences in connectivity between both the VST and vmPFC seeds and lateral regions of the middle frontal cortices. Specifically, children/adolescents with DBDs showed significantly less positive connectivity between the seeds and these regions in response to reward relative to non-reward, compared to healthy children/adolescents. Healthy children/adolescents show substantial connectivity between these regions potentially allowing reinforcement information to modulate attention as well as top down control over emotional responding. ${ }^{51)}$ Notably, previous work has reported that both adolescent smokers and adolescents with ADHD show increased connectivity relative to comparison adolescents rather than the reduced positive connectivity in children/adolescents with DBDs compared to healthy children/adolescents seen here. ${ }^{52,53)}$ The current results suggest that even if children/adolescents with DBDs show a relatively intact VST/VmPFC response to reward, the degree to which this region interacts with cortical structures in charge of allocating attention and regulating emotional responding during reward processing is compromised. ${ }^{4}$ In addition, the children/adolescents with DBDs in the current study showed significantly reduced positive connectivity between the vmPFC seed and the amygdala ROI for non-reward relative to reward. This is consistent with several previous findings of reduced VmPFC-amygdala connectivity in children/adolescents with DBDs. ${ }^{28,54,56,57)} \mathrm{It}$ is argued that this reflects impairment in the integrated func- tioning of these regions during reinforcement-based (particularly punishment-based) decision-making in children/adolescents with DBDs. ${ }^{6}$

Three caveats should be considered with respect to the current data. First, while we included children/adolescents with comorbid ADHD and substance abuse, follow-up analyses excluding these children/adolescents revealed similar results with respect to the main findings (Supplementary Section 3, 4). Second, only stimulant medications were withheld for at least 48 hours before the fMRI procedures. Once again, however, follow-up analyses excluding these participants yielded comparable results with respect to the main findings (Supplementary Material). Third, we did not correlate symptoms of DBD with BOLD response. This warrants more comprehensive future study.

In conclusion, considerable previous work has suggested that children/adolescents with DBDs show reduced responsiveness to non-social rewards ${ }^{2,3,5)}$ and reduced modulation of their behavior as a function of other individual's distress cues. ${ }^{29)}$ With respect to the modulation of behavior by distress cues, the current data expand previous work by demonstrating that regions implicated in reinforcement-based decision-making (PCC and caudate) show significant differences in responses to distress cues in children/adolescents with DBDs relative to healthy children/adolescents in the context of an instrumental learning task. In addition, the current functional connectivity findings are suggestive that children/adolescents with DBDs may face difficulty in the allocation of attention during reinforcement-based decision-making.

\section{- Acknowledgments}

Data collection for this study was supported by the Intramural Research Program at the National Institute of Mental Health, National Institutes of Health under grant number 1-ZIA-MH002860-08 to Dr. Blair. In addition, this work was supported by 1-K22 MH109558-01. None of the authors has conflict of interest in regard to this study.

\section{SUPPLEMENTARY MATERIALS}

Supplementary data is available at https://doi.org/10.9758/cpn.2018.16.4.449. 


\section{REFERENCES}

1. American Psychiatric Association. Diagnostic and Statistical Manual of Mental Disorders: DSM-5. Washington D.C.: American Psychiatric Association;2013.

2. White SF, Pope K, Sinclair S, Fowler KA, Brislin SJ, Williams WC, et al. Disrupted expected value and prediction error signaling in youths with disruptive behavior disorders during a passive avoidance task. Am J Psychiatry 2013;170:315-323.

3. White SF, Tyler PM, Erway AK, Botkin ML, Kolli V, Meffert $\mathrm{H}$, et al. Dysfunctional representation of expected value is associated with reinforcement-based decision-making deficits in adolescents with conduct problems. J Child Psychol Psychiatry 2016;57:938-946.

4. Matthys W, Vanderschuren LJ, Schutter DJ, Lochman JE. Impaired neurocognitive functions affect social learning processes in oppositional defiant disorder and conduct disorder: implications for interventions. Clin Child Fam Psychol Rev 2012;15:234-246.

5. Crowley TJ, Dalwani MS, Mikulich-Gilbertson SK, Du YP, Lejuez CW, Raymond KM, et al. Risky decisions and their consequences: neural processing by boys with antisocial substance disorder. PLoS One 2010;5:e12835.

6. Blair RJ, Leibenluft E, Pine DS. Conduct disorder and callous-unemotional traits in youth. N Engl J Med 2014;371: 23:2207-2216.

7. Clithero JA, Rangel A. Informatic parcellation of the network involved in the computation of subjective value. Soc Cogn Affect Neurosci 2014;9:1289-1302.

8. Ernst M, Paulus MP. Neurobiology of decision making: a selective review from a neurocognitive and clinical perspective. Biol Psychiatry 2005;58:597-604.

9. McClure SM, York MK, Montague PR. The neural substrates of reward processing in humans: the modern role of FMRI. Neuroscientist 2004; 10:260-268.

10. Lin A, Adolphs R, Rangel A. Social and monetary reward learning engage overlapping neural substrates. Soc Cogn Affect Neurosci 2012;7:274-281.

11. Chang SW, Fagan NA, Toda K, Utevsky AV, Pearson JM, Platt ML. Neural mechanisms of social decision-making in the primate amygdala. Proc Natl Acad Sci U S A 2015;112: 16012-16017.

12. Meder D, Madsen KH, Hulme O, Siebner HR. Chasing probabilities - Signaling negative and positive prediction errors across domains. Neuroimage 2016;134:180-191.

13. Budhani S, Marsh AA, Pine DS, Blair RJ. Neural correlates of response reversal: considering acquisition. Neuroimage 2007; 34:1754-1765.

14. Casey BJ, Forman SD, Franzen P, Berkowitz A, Braver TS, Nystrom LE, et al. Sensitivity of prefrontal cortex to changes in target probability: a functional MRI study. Hum Brain Mapp 2001;13:26-33.

15. Liu X, Powell DK, Wang H, Gold BT, Corbly CR, Joseph JE.
Functional dissociation in frontal and striatal areas for processing of positive and negative reward information. J Neurosci 2007;27:4587-4597.

16. Kuhnen CM, Knutson B. The neural basis of financial risk taking. Neuron 2005;47:763-770.

17. Finger EC, Marsh AA, Mitchell DG, Reid ME, Sims C, Budhani $\mathrm{S}$, et al. Abnormal ventromedial prefrontal cortex function in children with psychopathic traits during reversal learning. Arch Gen Psychiatry 2008;65:586-594.

18. White SF, Fowler KA, Sinclair S, Schechter JC, Majestic CM, Pine DS, et al. Disrupted expected value signaling in youth with disruptive behavior disorders to environmental reinforcers. J Am Acad Child Adolesc Psychiatry 2014;53:579-588.e9.

19. Rubia K, Smith AB, Halari R, Matsukura F, Mohammad M, Taylor $\mathrm{E}$, et al. Disorder-specific dissociation of orbitofrontal dysfunction in boys with pure conduct disorder during reward and ventrolateral prefrontal dysfunction in boys with pure ADHD during sustained attention. Am J Psychiatry 2009;166: 83-94.

20. Finger EC, Marsh AA, Blair KS, Reid ME, Sims C, Ng P, et al. Disrupted reinforcement signaling in the orbitofrontal cortex and caudate in youths with conduct disorder or oppositional defiant disorder and a high level of psychopathic traits. Am J Psychiatry 2011;168:152-162.

21. Blakemore SJ. The social brain in adolescence. Nat Rev Neurosci 2008;9:267-277.

22. Scott-Van Zeeland AA, Dapretto M, Ghahremani DG, Poldrack RA, Bookheimer SY. Reward processing in autism. Autism Res 2010;3:53-67.

23. Blair RJ, White SF, Meffert H, Hwang S. Emotional learning and the development of differential moralities: implications from research on psychopathy. Ann N Y Acad Sci 2013;1299: 36-41.

24. Averill JR. Anger and aggression: an essay on emotion. New York:Springer-Verlag; 1982.

25. Blair RJ. Facial expressions, their communicatory functions and neuro-cognitive substrates. Philos Trans R Soc Lond B Biol Sci 2003;358:561-572.

26. Dawel A, O'Kearney R, McKone E, Palermo R. Not just fear and sadness: meta-analytic evidence of pervasive emotion recognition deficits for facial and vocal expressions in psychopathy. Neurosci Biobehav Rev 2012;36:2288-2304.

27. Marsh AA, Blair RJ. Deficits in facial affect recognition among antisocial populations: a meta-analysis. Neurosci Biobehav Rev 2008;32:454-465.

28. Marsh AA, Finger EC, Mitchell DG, Reid ME, Sims C, Kosson DS, et al. Reduced amygdala response to fearful expressions in children and adolescents with callous-unemotional traits and disruptive behavior disorders. Am J Psychiatry 2008; 165: 712-720.

29. Blair RJ, Colledge E, Murray L, Mitchell DG. A selective impairment in the processing of sad and fearful expressions in children with psychopathic tendencies. I Abnorm Child 
Psychol 2001;29:491-498.

30. White SF, Marsh AA, Fowler KA, Schechter JC, Adalio C, Pope $\mathrm{K}$, et al. Reduced amygdala response in youths with disruptive behavior disorders and psychopathic traits: decreased emotional response versus increased top-down attention to nonemotional features. Am J Psychiatry 2012;169:750-758.

31. Lozier LM, Cardinale EM, VanMeter JW, Marsh AA. Mediation of the relationship between callous-unemotional traits and proactive aggression by amygdala response to fear among children with conduct problems. JAMA Psychiatry 2014;71:627-636.

32. Blair RJ, White SF, Meffert H, Hwang S. Disruptive behavior disorders: taking an RDoC(ish) approach. Curr Top Behav Neurosci 2014;16:319-336.

33. Insel T, Cuthbert B, Garvey M, Heinssen R, Pine DS, Quinn K, et al. Research domain criteria (RDoC): toward a new classification framework for research on mental disorders. Am J Psychiatry 2010;167:748-751.

34. Kaufman J, Birmaher B, Brent D, Rao U, Flynn C, Moreci P, et al. Schedule for Affective Disorders and Schizophrenia for School-age Children-Present and Lifetime version (K-SADS-PL): Initial reliability and validity data. I Am Acad Child AdolesC Psychiatry 1997;36:980-988.

35. Wechsler D. Wechsler abbreviated scale of intelligence. San Antonio, TX.:Psychological Corporation; 1999.

36. Talairach J, Tournoux P. Co-planar stereotaxic atlas of the human brain: an approch to cerebral imaging. Stuttgart:Thieme; 1988.

37. Lieberman MD, Cunningham WA. Type / and Type // error concerns in $\mathrm{AMRI}$ research: re-balancing the scale. Soc Cogn Affect Neurosci 2009;4:423-428.

38. Eklund A, Nichols TE, Knutsson H. Cluster failure: Why fMRI inferences for spatial extent have inflated false-positive rates. Proc Natl Acad Sci U S A 2016;113:7900-7905.

39. McLaren DG, Ries ML, Xu G, Johnson SC. A generalized form of context-dependent psychophysiological interactions (gPPl): a comparison to standard approaches. Neuroimage 2012;61:1277-1286.

40. Hwang S, Nolan ZT, White SF, Williams WC, Sinclair S, Blair RJ. Dual neurocircuitry dysfunctions in disruptive behavior disorders: emotional responding and response inhibition. Psychol Med 2016;46:1485-1496.

41. Motzkin JC, Newman JP, Kiehl KA, Koenigs M. Reduced prefrontal connectivity in psychopathy. I Neurosci 2011;31: 17348-17357.

42. Frick PJ. The inventory of callous-unemotional traits. New Orleans:University of New Orleans;2004.

43. Silverman MH, Jedd K, Luciana M. Neural networks involved in adolescent reward processing: an activation likelihood estimation meta-analysis of functional neuroimaging studies. Neuroimage 2015; 122:427-439.
44. O’Doherty J, Dayan P, Schultz J, Deichmann R, Friston K, Dolan RJ. Dissociable roles of ventral and dorsal striatum in instrumental conditioning. Science 2004;304:452-454.

45. Wendler E, Gaspar JC, Ferreira TL, Barbiero JK, Andreatini R, Vital MA, et al. The roles of the nucleus accumbens core, dorsomedial striatum, and dorsolateral striatum in learning: performance and extinction of Pavlovian fear-conditioned responses and instrumental avoidance responses. Neurobiol Learn Mem 2014;109:27-36.

46. Wrase J, Kahnt T, Schlagenhauf F, Beck A, Cohen MX, Knutson B, et al. Different neural systems adjust motor behavior in response to reward and punishment. Neuroimage 2007; 36:1253-1262.

47. Blair RJ, Jones L, Clark F, Smith M. The psychopathic individual: a lack of responsiveness to distress cues? Psychophysiology 1997;34:192-198.

48. Passamonti L, Fairchild G, Goodyer IM, Hurford G, Hagan CC, Rowe JB, et al. Neural abnormalities in early-onset and adolescence-onset conduct disorder. Arch Gen Psychiatry 2010;67:729-738.

49. Blair RJ. The amygdala and ventromedial prefrontal cortex in morality and psychopathy. Trends Cogn Sci 2007;11:387392.

50. Blair RJ. A cognitive developmental approach to mortality: investigating the psychopath. Cognition 1995;57:1-29.

51. Haber SN, Knutson B. The reward circuit: linking primate anatomy and human imaging. Neuropsychopharmacology 2010;35:4-26.

52. Ma I, van Holstein M, Mies GW, Mennes M, Buitelaar J, Cools $\mathrm{R}$, et al. Ventral striatal hyperconnectivity during rewarded interference control in adolescents with ADHD. Cortex 2016; 82:225-236.

53. Jollans L, Zhipeng C, Icke I, Greene C, Kelly C, Banaschewski $\mathrm{T}$, et al. Ventral striatum connectivity during reward anticipation in adolescent smokers. Dev Neuropsychol 2016;41: 6-21.

54. Finger EC, Marsh A, Blair KS, Majestic C, Evangelou I, Gupta $\mathrm{K}$, et al. Impaired functional but preserved structural connectivity in limbic white matter tracts in youth with conduct disorder or oppositional defiant disorder plus psychopathic traits. Psychiatry Res 2012;202:239-244.

55. Marsh AA, Finger EC, Fowler KA, Jurkowitz ITN, Schechter JC, $\mathrm{Yu} \mathrm{HH}$, et al. Reduced amygdala-orbitofrontal connectivity during moral judgments in youths with disruptive behavior disorders and psychopathic traits. Psychiatry Res 2011;194: 279-286.

56. Aghajani M, Klapwijk ET, van der Wee NJ, Veer IM, Rombouts $\mathrm{SARB}$, Boon AE, et al. Disorganized amygdala networks in conduct-disordered juvenile offenders with callous-unemotional traits. Biol Psychiatry 2017;82:283-293. 